\title{
Randomized Algorithms for Probabilistic Aircraft Conflict Detection*
}

\author{
Maria Prandini, John Lygeros, Arnab Nilim and Shankar Sastry \\ Department of Electrical Engineering and Computer Sciences \\ University of California, Berkeley CA 94720 \\ \{prandini, lygeros, nilim, sastry\}@robotics.eecs . berkeley.edu
}

\begin{abstract}
A mid-range conflict alerting system is proposed, based on a measure of criticality which directly takes into account the uncertainty in the prediction of the aircraft positions. The use of randomized algorithms makes the computation of the criticality measure tractable. The performance of the algorithm is evaluated by Monte Carlo simulation on a stochastic ODE model of the aircraft motion.
\end{abstract}

\section{Introduction}

The increasing demand for air travel is stressing the current, mostly human operated, Air Traffic Management System (ATMS). It has been suggested that an increase in the level of automation in future ATMS may alleviate some of this stress, by improving the efficiency of the system and simplifying the tasks of the human operators, while improving - or at least maintaining - the current level of safety. Safety is typically quantified in terms of numbers of conflicts, that is situations where aircraft come closer to one another than a certain desired minimum distance. To prevent conflicts an automated ATMS has to be able to perform conflict detection (predict the position of aircraft in the future to determine if conflicts are possible) and conflict resolution (modify the plans of the aircraft to prevent the conflicts). Currently, these functions are performed manually by the pilots and air traffic controllers (ATC), sometimes with the assistance of partial automation tools (for example CTAS [1] and TCAS [2]).

Conflict detection and resolution schemes can be classified into long range $([3,4])$, mid-range $([5,6,7])$, and short range $([2,6,7])$, depending on the time horizon over which prediction is performed. In this paper, we concentrate on mid-range conflict prediction, over horizons of the order of tens of minutes. Currently this

${ }^{*}$ Research supported by DARPA under F33615-98-C-3614, by NASA under NAG 2-1039 and by ARO under DAAH 04-96-10341. The authors would like to thank Dr. George J. Pappas and Jianghai $\mathrm{Hu}$ for stimulating discussions. function is carried out by ATC. The prediction algorithm proposed here is based on a probabilistic model for aircraft motion introduced in [5]. The model provides probability distributions for the future positions of the aircraft, based on their current positions and their flight plans. The distributions are used to compute the maximum probability of conflict over a 20 minutes horizon. If the probability exceeds a certain threshold a conflict is declared. Computational complexity and the requirement for real time implementation make it difficult to directly apply this procedure in practice. To keep the problem computationally tractable, we use randomized algorithms to obtain an estimate of the probability of conflict, as well as theoretical bounds on the level of the approximation involved.

The performance of our algorithm is evaluated by Monte Carlo simulation. Since the prediction model is somewhat inaccurate, the simulation is based on a more detailed, stochastic ODE model for the motion of the aircraft. The simulation results are also used to tune the parameters of the algorithm, in an attempt to optimize the tradeoff between probability of successful conflict detection and probability of false alarm.

\section{Probabilistic Models}

\subsection{Flight Plans and Configuration}

Consider $N_{a}$ aircraft sharing a region of the airspace. Assume that the flight plan of aircraft $i$ consists of a sequence of way points, $\left\{P_{j}^{i}\right\}_{j=0}^{n_{i}}, P_{j}^{i} \in \mathbb{R}^{3}$, given in a global coordinate frame, and a sequence of speeds, $\left\{v_{j}^{i}\right\}_{j=1}^{n_{i}}, v_{j}^{i} \in \mathbb{R}_{+}$. The interpretation is that aircraft $i$ follows the sequence of way points, moving roughly along the straight line joining successive way points $P_{j-1}^{i}$ and $P_{j}^{i}$ with velocity $v_{j}^{i} . P_{0}^{i}$ and $P_{n_{i}}^{i}$ respectively represent the current position of aircraft $i$ (available through ADSB or radar) and the threshold of the landing runway at the destination airport. The nominal time of arrival of aircraft $i$ at way point $j$ can be recursively computed by $T_{j}^{i}=\left\|P_{j}^{i}-P_{j-1}^{i}\right\| / v_{j}^{i}+T_{j-1}^{i}, j>0$, 
with $T_{0}^{i}=0$. Likewise, the nominal distance traveled by aircraft $i, s^{i}(t) \in \mathbb{R}$, and its nominal position, $p^{i}(t) \in \mathbb{R}^{3}$, at time $t \in\left(T_{j-1}^{i}, T_{j}^{i}\right]$, can be respectively computed by $s^{i}\left(T_{0}^{i}\right)=0, s^{i}(t)=v_{j}^{i}\left(t-T_{j-1}^{i}\right)+s^{i}\left(T_{j-1}^{i}\right)$, and $p^{i}(t)=P_{j-1}^{i}+v_{j}^{i}\left(t-T_{j-1}^{i}\right) \frac{P_{j}^{i}-P_{j-1}^{i}}{\left\|P_{j}^{i}-P_{j-1}^{i}\right\|}$.

The configuration, $\gamma$, of the $N_{a}$ aircraft system consists of the flight plans of all aircraft, $\gamma=$ $\left\{\left\{P_{j}^{i}\right\}_{j=0}^{n_{i}},\left\{v_{j}^{i}\right\}_{j=1}^{n_{i}}\right\}_{i=1}^{N_{a}}$. We assume that the flight plan of each aircraft is known, except of course the way point encoding the current position, which depends on how well the aircraft is tracking the flight plan. The models introduced below assume that an aircraft turns instantaneously and heads for the new way point at the scheduled time, even if it has deviated from its flight plan. This assumption is somewhat unrealistic, but is used in the prediction model to simplify the computation. It can be easily relaxed for the validation model, if better models for the way aircraft execute turns become available.

\subsection{Prediction model}

The actual position of the aircraft is affected by uncertainty, due to wind and errors in tracking, navigation, and control. Following $[5,6]$, we assume that the predicted position $x^{i}(t) \in \mathbb{R}^{3}$, of aircraft $i$ can be modeled as a multivariate Gaussian random variable, $x^{i}(t) \sim \mathcal{N}\left(p^{i}(t), Q^{i}(t)\right)$, independent of the random variables modeling the positions of other aircraft. Notice that the mean is equal to the nominal position of aircraft $i$ along its flight plan. The variance of the predicted position is assumed to increase with time, reflecting the fact that the uncertainty about the position of the aircraft increases the further we try to predict into the future. In [5], a distinction is made between the variances in the along track and cross track directions. It is assumed that the standard deviation of the along track component grows linearly with time:

$$
\sigma_{A T}^{i}(t)=c_{1}+c_{2} t
$$

The standard deviation of the horizontal cross track component, grows linearly with the distance traveled and then saturates at a fixed value:

$$
\sigma_{C T H}^{i}(t)=\min \left\{c_{4}, c_{1}+c_{3} s^{i}(t)\right\}
$$

Finally, the standard deviation of the vertical cross track component, remains constant:

$$
\sigma_{C T V}^{i}(t)=c_{5}
$$

The values $c_{1}=50 / 1850 \mathrm{nmi}, c_{2}=0.25 \mathrm{nmi} / \mathrm{min}$, $c_{3}=1 / 57, c_{4}=1 \mathrm{nmi}$, and $c_{5}=30 / 1850 \mathrm{nmi}$ were proposed in $[5,6]$, based on empirical air traffic data. Since the uncertainty components are assumed to be independent, the covariance matrix for $t \in\left[T_{j-1}^{i}, T_{j}^{i}\right)$ is given by:

$$
Q^{i}(t)=R\left[\begin{array}{ccc}
\left(\sigma_{A T}^{i}\right)^{2}(t) & 0 & 0 \\
0 & \left(\sigma_{C T H}^{i}\right)^{2}(t) & 0 \\
0 & 0 & \left(\sigma_{C T V}^{i}\right)^{2}(t)
\end{array}\right] R^{T}
$$

where we set $R \in S O(3)$ for the rotation matrix $R\left(\theta_{j}^{i}, \phi_{j}^{i}\right)$ associated with the angles $\theta_{j}^{i}$ and $\pi / 2-\phi_{j}^{i}$ that the vector $P_{j}^{i}-P_{j-1}^{i}$ makes with the $x_{1}$ and $x_{3}$ axes of the global coordinate frame in which the $P_{j}^{i}$ s are given.

This model is fairly accurate for mid-range conflict prediction, as it reflects the fact that pilots tend to correct cross track errors in the short term and deal with along track errors in the long term, using small changes in speed [5]. The accuracy of the model is limited by the assumption that the positions of the aircraft are uncorrelated. Since the tracking noise is primarily due to wind, the positions of the aircraft may in fact be correlated, especially near the conflict point where they are close one to the other. We are currently investigating ways of relaxing this assumption.

\subsection{Validation model}

The prediction model is simple and allows fast computations, but has inherent limitations, which limit its applicability for simulation and validation. To remove some of these limitations we introduce a stochastic ODE model and use it to generate aircraft trajectories for validation. The validation model provides a formal way of correlating the positions of a single aircraft at different points in time.

Consider an aircraft moving in $\mathbb{R}^{3}$, and let $x$ denote its position with respect to a global inertial coordinate frame. Assume its velocity has magnitude $v$ and makes an angle $\theta$ with respect to the $x_{1}$ axis and $\pi / 2-\phi$ with respect to the $x_{3}$ axis. Consider a body coordinate frame $\chi=\left[\begin{array}{lll}\chi_{1} & \chi_{2} & \chi_{3}\end{array}\right]^{T}$ with $\chi_{1}$ aligned with the aircraft velocity (along track), $\chi_{2}$ perpendicular to it and lying on the plane on which the aircraft flies (cross track horizontal), and $\chi_{3}$ perpendicular to $\chi_{1}$ and $\chi_{2}$ (cross track vertical) (Figure 1). The two frames are related through the coordinate transformation

$$
x=R(\theta, \phi) \chi+p,
$$

where $R(\theta, \phi)$ is the rotation matrix and $p$ denotes the position of the origin of the body frame with respect to the inertial frame. $p$ can be interpreted as the nominal position of the aircraft, $x$ as its actual position, and $\chi$ as the variation of the aircraft position with respect to the nominal one along the tracking directions.

The nominal position $p$ evolves according to:

$$
\dot{p}=R(\theta, \phi)\left[\begin{array}{lll}
1 & 0 & 0
\end{array}\right]^{T} v
$$




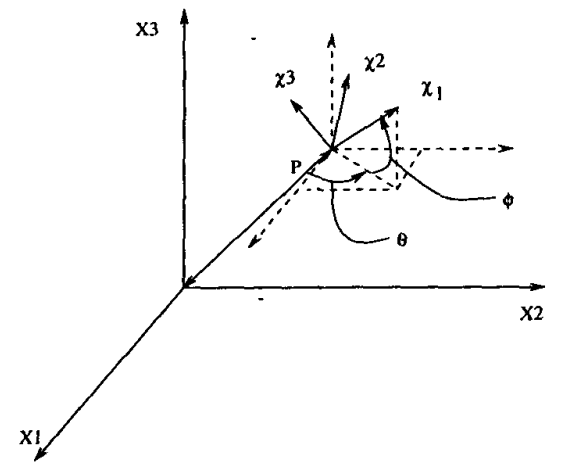

Figure 1: The body and inertial frames.

We assume that the uncertainty in the position around the nominal point is obtained through the ODE:

$$
\dot{\chi}=A \chi+\eta
$$

where $\chi(0) \sim \mathcal{N}\left(0, V_{\chi}(0)\right)$ and $\eta \in \mathbb{R}^{3}$ is a white Gaussian noise $\eta(t) \sim \mathcal{N}\left(0, V_{\eta}(t)\right)$ independent of $\chi(0)$. Combining equations (1)-(3) produces the kinematic model

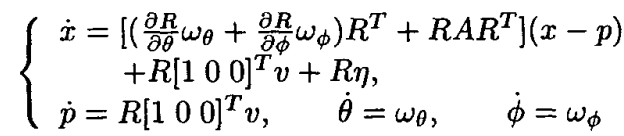

where $\left(v, \omega_{\theta}, \omega_{\phi}\right)$ are the linear and angular velocities.

The above equations describing the aircraft motion are nonlinear. To make them linear we can adopt the simplification $\omega_{\theta} \equiv \omega_{\phi} \equiv 0$, and model turns as discrete events occurring at the way points. This leads to a piecewise linear stochastic ODE: for $t \in\left[T_{j-1}, T_{j}\right)$,

$$
\left\{\begin{array}{l}
\dot{x}=A\left(\theta_{j}, \phi_{j}\right)(x-p)+B\left(\theta_{j}, \phi_{j}\right) v_{j}+C\left(\theta_{j}, \phi_{j}\right) \eta \\
\dot{p}=D\left(\theta_{j}, \phi_{j}\right) v_{j}
\end{array}\right.
$$

where $A(\theta, \phi)=R A R^{T}, C(\theta, \phi)=R$, and $B(\theta, \phi)=$ $D(\theta, \phi)=R\left[\begin{array}{lll}1 & 0 & 0\end{array}\right]^{T}$. The initial conditions are $p(0)=$ $P_{0}$ and $x(0) \sim \mathcal{N}\left(P_{0}, R\left(\theta_{1}, \phi_{1}\right) V_{\chi}(0) R\left(\theta_{1}, \phi_{1}\right)^{T}\right)$, independent of the white Gaussian noise $\eta \sim \mathcal{N}\left(0, V_{\eta}(t)\right)$. The initial conditions and the noise processes for different aircraft are assumed to be independent.

Position estimates $y \in \mathbb{R}^{3}$ for each aircraft are obtained every $\Delta$ seconds through radar measurements (typically $\Delta=12$ ). We set

$$
y(k \Delta)=x(k \Delta)+\xi(k \Delta),
$$

where the noise $\{\xi(k \Delta)\}_{k \geq 0}$ is described as a sequence of i.i.d. Gaussian random variables with $\xi(k \Delta) \sim$ $\mathcal{N}\left(0, V_{\xi}\right)$ and it is independent of all the other random variables involved in the validation model.

For our stochastic validation model to resemble the statistics derived from air traffic data, we need to appropriately choose $V_{\chi}(0), V_{\eta}(t), A$ and $V_{\xi}$. For the radar model parameters, we set $V_{\xi}=\operatorname{diag}\left(V_{\xi_{2}}, V_{\xi_{2}}, V_{\xi_{3}}\right)$ where $V_{\xi_{1}}=V_{\xi_{2}}=c_{1}^{2}$ and $V_{\xi_{3}}=c_{5}^{2}$, to reproduce the statistical characteristics described in [5] for the uncertainty in the current aircraft position. For the aircraft model we set $V_{\chi_{1}}(0)=V_{\chi_{2}}(0)=c_{1}^{2}, V_{\chi_{3}}(0)=c_{5}^{2}$, $V_{\eta_{1}}(t)=2 c_{1} c_{2}+2 c_{2}^{2} t, V_{\eta_{2}}=2 a_{2} c_{4}^{2}, V_{\eta_{3}}=2 a_{3} c_{5}^{2}$, where $A=\operatorname{diag}\left(0,-a_{2},-a_{3}\right), a_{2}=\frac{c_{3}}{\left(c_{4}-c_{1}\right)} v_{1}$ and $a_{3}=1 n m i^{-1}$. From standard results in stochastic linear systems [8], $\chi_{1}(t), \chi_{2}(t)$, and $\chi_{3}(t)$ will then be independent, zero mean Gaussian random variables, with

$$
\begin{aligned}
\operatorname{var}\left[\chi_{1}(t)\right] & =\left(c_{1}+c_{2} t\right)^{2} \\
\operatorname{var}\left[\chi_{2}(t)\right] & =c_{4}^{2}+\left[c_{1}^{2}-c_{4}^{2}\right] e^{-\frac{2 c_{3}}{\left(c_{4}-c_{1}\right)} v_{1} t} \\
\operatorname{var}\left[\chi_{3}(t)\right] & =c_{5}^{2}
\end{aligned}
$$

$\chi_{1}$ and $\chi_{3}$ are Gaussian processes exactly matching the mean and variance characteristics of the along track and vertical cross track errors. The time constant of the exponential for the Gaussian process $\chi_{2}$ is equal to $1 / 2$ of the time $\bar{t}=\frac{c_{4}-c_{1}}{c_{3} v_{1}}$ required for $\sigma_{C T H}^{2}(t)$ to reach the saturation value $c_{4}^{2}$ in the case when the aircraft trajectory is a straight line traveled with speed $v_{1}$. For typical speeds $\left(v_{1}=500 n m i / h\right), \operatorname{var}\left[\chi_{2}(t)\right]$ reaches $86 \%$ of the saturation value in about 6 minutes. Ideally, the value of $a_{3}$ should be set based on the estimate of the correlation between the aircraft positions at different time instants, as it does not affect any other relevant statistics. Since this piece of information is not available in the literature, we arbitrarily set it equal to $1 n m i^{-1}$.

\section{Conflict Detection}

\subsection{General Conflict Detection}

The desired separation among aircraft is typically encoded by means of a minimum horizontal separation, $d_{H}$, and a minimum vertical separation, $d_{V}$. Currently $d_{H}=5 n m i$ for enroute airspace and $3 n m i$ in the TRACON, and $d_{V}=2000 \mathrm{ft}$ above $29000 \mathrm{ft}$ and $1000 \mathrm{ft}$ below $29000 \mathrm{ft}$. The conflict set is then given by $\mathcal{C}=$ $\left\{\left(u_{1}, u_{2}, u_{3}\right) \in \mathbb{R}^{3}:\left(u_{1}^{2}+u_{2}^{2} \leq d_{H}^{2}\right) \wedge\left(\left|u_{3}\right| \leq d_{V}\right)\right\}$

Conflict detection consists of extracting some measure, $C(\gamma)$, of how safety critical the current configuration, $\gamma$, is, comparing this measure to a threshold, $P$, and declaring a conflict if $P$ is exceeded. The process should be repeated every time $\gamma$ changes, that is when a new measurement comes in from the radar, the ATC changes a flight plan, etc.

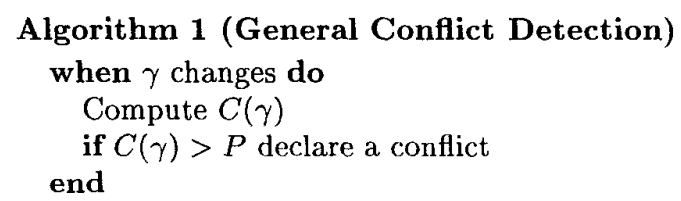


In this paper we use the maximum of the probability of conflict over a horizon $T$ as a measure of criticality* Consider two aircraft, 1 and 2, let $x^{1}$ and $x^{2}$ denote their positions in the inertial reference frame. Given the probability density function $f(u, t)$ for the separation $u(t)=x^{1}(t)-x^{2}(t)$ of the two aircraft at time $t$, the probability of conflict at time $t$ is given by

$$
P C(t)=\operatorname{Prob}(u \in \mathcal{C})=\int_{\mathcal{C}} f(u, t) d u .
$$

For the prediction model of Section 2.2, the predicted separation of two aircraft at time $t$ is a Gaussian random variable with mean $\mu(t)=p^{1}(t)-p^{2}(t)$ and covariance matrix $Q(t)=Q^{1}(t)+Q^{2}(t)$. We set $C(\gamma)=$ $\sup _{t \in[0, T]} P C(t)$, and, following [5], $T=20$ minutes.

The major obstacle in the implementation of Algorithm 1 is the computation of $C(\gamma)$, since one can not derive an analytical expression for $P C(\cdot)$. In the literature techniques for estimating $C(\gamma)$ have been proposed using Monte Carlo simulation [6] and analytical approximation [5]. We propose an algorithm for approximating $C(\gamma)$ based on the theory of empirical processes [9].

\subsection{Estimation of $C(\gamma)$}

Suppose for the time being that we are able to compute $P C(t)$ with no error. Let $\mathcal{Q}$ be the uniform distribution on $[0, T]$, and consider the following algorithm for computing an estimate, $C^{\prime}(\gamma)$, of $C(\gamma)$.

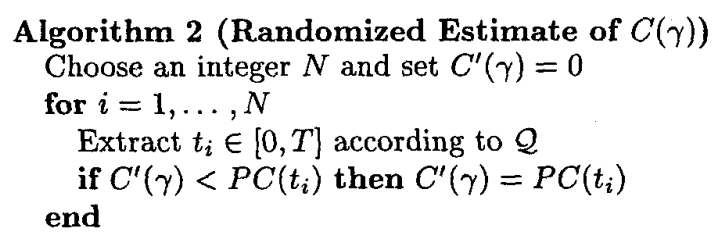

Clearly, $C^{\prime}(\gamma)=\max _{t \in\left\{t_{i}\right\}_{i=1}^{N}} P C(t) \leq C(\gamma)$, since we are testing just $N$ values of $P C(t)$. In addition, the quality of the approximation is random due to the stochastic selection of the $t_{i}$ 's. Nevertheless, if the random extractions are independent, it can be shown that $C^{\prime}$ is a good approximation in a probabilistic sense.

Theorem 1 (Estimation of $C(\gamma)$ ) Fix $\beta>0$ and consider the probability space $([0, T], \mathcal{F}, \mathcal{Q})$ where $\mathcal{F}$ is the Borel $\sigma$-algebra on $[0, T]$. Then

$$
\mathcal{Q}\left\{t \in[0, T]: P C(t)>C^{\prime}(\gamma)\right\} \leq \beta
$$

with probability greater than $1-(1-\beta)^{N}$.

In other words, if we set $N=\left\lceil\frac{\ln (\delta)}{\ln (1-\beta)}\right\rceil$ (where $\lceil z\rceil$ denotes the smallest integer greater than $z$ ), there exists

\footnotetext{
*Other measures of criticality (such as weighted averages of the probability of conflict) were also tested as part of this study and were found to be less effective than the maximum.
}

an exceptional $S \subset[0, T]$ of Lebesgue measure at most $\beta T$ such that $\sup _{[0, T] \backslash S} P C(t) \leq C^{\prime} \leq \sup _{[0, T]} P C(t)$ with probability at least $1-\delta$. Hence, $C^{\prime}$ is an approximation of $C$ in the sense that is bracketed by the supremum of $P C(t)$ over all $[0, T]$ and the supremum of $P C(t)$ over "nearly" all of $[0, T]$ with high probability.

\subsection{Estimation of $P C(t)$}

Next, we introduce a method for computing a uniformly good approximation of $P C(t)$ over a finite set of time instants $\left\{t_{1}, t_{2}, \ldots, t_{N}\right\}$. Recall that $P C(t)$ is the measure of the fixed set $\mathcal{C}$ according to $\mathcal{N}(\mu(t), Q(t))$. By an appropriate change of coordinates, however, it can also be viewed as the measure of a time dependent set, $\mathcal{C}_{t}$, according to the standard normal distribution $\mathcal{N}(0, I)$. The required change of coordinates can be found by computing the Cholesky factorization $^{\dagger} Q(t)=L(t) L(t)^{T}$ of the covariance matrix, and setting $w=L(t)^{-1}[u-\mu(t)]$. We then get $P C(t)=\int_{\mathcal{C}_{t}} \frac{1}{2 \pi} e^{-\frac{1}{2} w^{T} w} d w$, where $\mathcal{C}_{t}=\left\{w \in \mathbb{R}^{3}:\right.$ $L(t) w+\mu(t) \in \mathcal{C}\}$. This suggests the following algorithm for probabilistically estimating $P C(t)$.

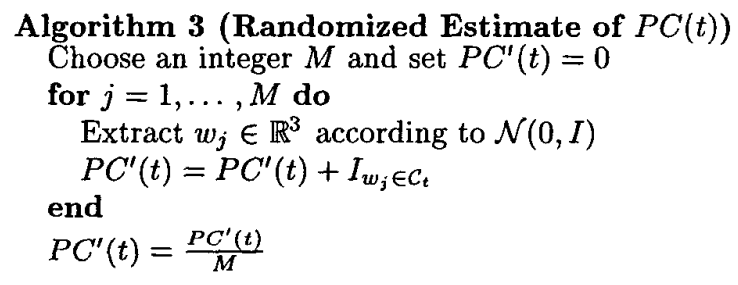

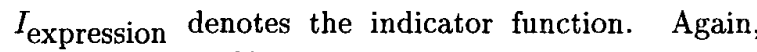
$P C^{\prime}(t)=\frac{1}{M} \sum_{j=1}^{M} I_{w_{j} \in \mathcal{C}_{t}}$ is a random approximation of $P C(t)$, due to the stochastic selection of the $w_{j}$ 's. Under the assumption that the random extractions are independent, the following result quantifies the level of approximation involved.

Theorem 2 (Estimation of $P C$ ) $F i x \in \in(0,1)$ and consider the probability space $\left(\mathbb{R}^{3}, \mathcal{F}, \mathcal{N}(0, I)\right)$ where $\mathcal{F}$ is the Borel $\sigma$-algebra on $\mathbb{R}^{3}$. Then,

$$
\begin{aligned}
& \mathcal{N}^{M}\left\{w^{M} \in \mathbb{R}^{3 M}: \sup _{t \in\left\{t_{i}\right\}_{i=1}^{N}}\left|P C^{\prime}\left(t_{i}\right)-P C\left(t_{i}\right)\right|>\epsilon\right\} \leq 2 N e^{-2 M \epsilon^{2}} \\
& \text { where } w^{M} \text { denotes }\left(w_{1}, w_{2}, \ldots, w_{M}\right)
\end{aligned}
$$

Hence, each finite collection of sets $\left\{\mathcal{C}_{t_{i}}\right\}_{i=1}^{N}$ has the property of uniform convergence of empirical probabilities since for each fixed $\epsilon$ the estimates uniformly convergences to their true values as the number of samples $M$ goes to infinity.

\subsection{Randomized Conflict Detection} The following algorithm brings the two procedures together.

\footnotetext{
${ }^{\dagger}$ A similar procedure is followed in [5].
} 


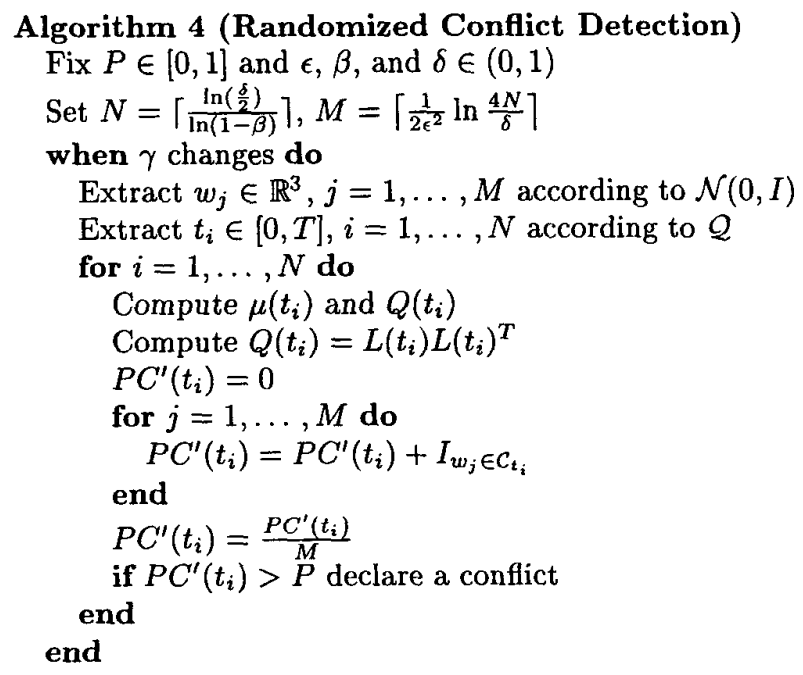

A conflict is declared if and only if the estimate

$$
C^{\prime}(\gamma)=\max _{i=1, \ldots, N} \frac{1}{M} \sum_{j=1}^{M} I_{w_{j} \in \mathcal{C}_{t_{i}}}
$$

exceeds the threshold $P$. Under the assumption that all the random extractions are independent, the following theorem characterizes the accuracy of our approximation.

Theorem 3 (Approximate estimation of $C(\gamma)$ ) Given $\epsilon, \beta$, and $\delta \in(0,1), C^{\prime}(\gamma)$ is an approximate estimate of $C(\gamma)$ to accuracy $2 \epsilon$ and level $\beta$ with confidence $1-\delta$ in the sense that

$$
\mathcal{Q}\left\{t \in[0 T]: P C(t)>C^{\prime}(\gamma)+2 \epsilon\right\} \leq \beta,
$$

with probability greater than $1-\delta$.

Notice that the number of samples needed to achieve a certain approximation level is independent of the nature of the sample space and of the probability distribution. In particular, the computational load does not significantly increase in the $3 \mathrm{D}$ case with respect to the $2 \mathrm{D}$ case. This is not the case if one resorts to numerical methods based on gridding or to approximate analytic methods such the one in [5].

\section{Validation and Tuning}

The performance of the conflict detection scheme was evaluated using a three step process:

1. Given the flight plans of two aircraft, generate trajectories and radar measurements over a 20 minutes horizon using the discretized version of the validation model.
2. For every value of the - discretized - threshold:

a. Execute the detection algorithm at every radar measurement time.

b. Compute the probability of false alarm $\mathrm{P}(\mathrm{FA})$ (fraction of declared conflicts that did not materialize) and the probability of successful alert $\mathrm{P}(\mathrm{SA})$ (fraction of conflicts declared at least 60 seconds before they occur).

3. Plot the System Operating Characteristic (SOC) curve and choose the optimal threshold $P$.

The SOC is a plot of $\mathrm{P}(\mathrm{SA})$ versus $\mathrm{P}(\mathrm{FA})$, parameterized by the threshold. In principle, the more the SOC curve approaches the point $(0,1)$, the better the performance of the system is likely to be. $P$ is typically chosen to correspond the point on the the SOC curve closest to $(0,1)$, in an attempt to achieve an "optimal" compromise between $\mathrm{P}(\mathrm{FA})$ and $\mathrm{P}(\mathrm{SA})[10$ ].

To ensure our results are reasonable, we compare the performance of our algorithm with the algorithm of [5], which is based on the same description of the uncertainty. The measure of criticality used by [5] is the probability of conflict at the point of the minimum nominal separation. An estimate for this quantity is computed for the $2 \mathrm{D}$ case by an analytical overapproximation of the integral of the probability density function for the separation. To compare the two algorithms we also restrict our algorithms to the $2 \mathrm{D}$ case, and to simple crossing encounters, parameterized by the path crossing angle $\phi(\mathrm{deg})$, the minimum nominal separation $d_{\min }(n m i)$, and the nominal time $t_{\min }$ (min) to $d_{\min }$. The speeds of the aircraft are kept fixed $\left(v_{1}=480 \mathrm{nmi} / \mathrm{h}\right.$ and $\left.v_{2}=500 \mathrm{nmi} / \mathrm{h}\right)$. Tables 1 and 2 summarize the values of $P(F A)$ and $P(S A)$ corresponding to the optimal threshold for different encounter situations computed by running the two detection algorithms $(\epsilon=0.05, \delta=0.1, \beta=0.05)$. Note that when the minimum nominal distance is $0 \mathrm{nmi}$, in some entries of Table 1 and $2 \mathrm{P}(\mathrm{SA})=1$, whereas the optimal $P(F A)$ is not defined. The reason is that for $d_{m i n}=0 \mathrm{nmi}$ there is almost always a conflict, therefore, the optimal threshold is determined by maximizing $\mathrm{P}(\mathrm{SA})$, and there are not enough samples to get a statistically significant estimate of $\mathrm{P}(\mathrm{FA})$.

Both algorithms give a similar value for $P(S A)$, but $\mathrm{P}(\mathrm{FA})$ is lower with our algorithm. The reason for this is that the measure of criticality used in [5] is an over approximation of the probability of conflict at $t_{m i n}$.

Different configurations lead to different SOC curves and therefore to different optimal thresholds. In [5] the threshold is set using heuristic arguments. We choose the threshold based on the optimal thresholds corresponding to Table 1 for the case of $d_{m i n}=5 \mathrm{nmi}$. This 


\begin{tabular}{|c|c|c|c|c|}
\hline \multirow{2}{*}{$\phi$} & \multirow{2}{*}{$d_{\min }$} & \multicolumn{3}{|c|}{$t_{\min }$} \\
\cline { 3 - 5 } & 0 & 8 & 12 & 20 \\
\hline 30 & 0 & $(\mathrm{~N} . \mathrm{D}) / 1.0$. & $(\mathrm{~N} . \mathrm{D}) / 1.0$. & $(\mathrm{~N} . \mathrm{D}) / 1.0$. \\
\hline 30 & 5 & $0.35 / 0.80$ & $0.20 / 0.71$ & $0.19 / 0.75$ \\
\hline 30 & 10 & $0.17 / 0.64$ & $0.17 / 0.68$ & $0.17 / 0.84$ \\
\hline 45 & 0 & $(\mathrm{~N} . \mathrm{D}) / 1.0$. & $(\mathrm{~N} . \mathrm{D}) / 1.0$. & $0.26 / 0.80$ \\
\hline 45 & 5 & $0.19 / 0.75$ & $0.22 / 80$ & $0.20 / 0.78$ \\
\hline 45 & 10 & $0.41 / 0.60$ & $0.16 / 0.77$ & $0.11 / 0.76$ \\
\hline 90 & 0 & $(\mathrm{~N} . \mathrm{D}) / 1.0$. & $(\mathrm{~N} . \mathrm{D}) / 1.0$. & $0.26 / 0.80$ \\
\hline 90 & 5 & $0.27 / 0.78$ & $0.13 / 0.83$ & $0.21 / 0.84$ \\
\hline 90 & 10 & $0.01 / 1.0$ & $0.21 / 0.80$ & $0.17 / 0.93$ \\
\hline
\end{tabular}

Table 1: $\mathrm{P}(\mathrm{FA}) / \mathrm{P}(\mathrm{SA})$ for our algorithm.

\begin{tabular}{|c|c|c|c|c|}
\hline & & \multicolumn{3}{|c|}{$t_{\min }$} \\
\cline { 3 - 5 }$\phi$ & $d_{\min }$ & 8 & 12 & 20 \\
\hline 30 & 0 & $(\mathrm{~N} . \mathrm{D}) / 1.0$. & $(\mathrm{~N} . \mathrm{D}) / 1.0$. & $(\mathrm{~N} . \mathrm{D}) / 1.0$ \\
\hline 30 & 5 & $0.61 / 0.76$ & $0.46 / 0.63$ & $0.28 / 0.79$ \\
\hline 30 & 10 & $0.45 / 0.64$ & $0.37 / 0.68$ & $0.21 / 0.84$ \\
\hline 45 & 0 & $(\mathrm{~N} . \mathrm{D}) / 1.0$. & $(\mathrm{~N} . \mathrm{D}) / 1.0$. & $0.43 / 0.84$ \\
\hline 45 & 5 & $0.70 / 0.76$ & $0.58 / 0.80$ & $0.31 / 0.83$ \\
\hline 45 & 10 & $0.48 / 0.47$ & $0.33 / 0.77$ & $0.14 / 0.76$ \\
\hline 90 & 0 & $(\mathrm{~N} . \mathrm{D}) / 1.0$. & $(\mathrm{~N} . \mathrm{D}) / 1.0$. & $0.42 / 0.84$ \\
\hline 90 & 5 & $0.76 / 0.79$ & $0.52 / 0.85$ & $0.31 / 0.84$ \\
\hline 90 & 10 & $0.33 / 1.0$ & $0.30 / 0.73$ & $0.23 / 0.93$ \\
\hline
\end{tabular}

Table 2: $\mathrm{P}(\mathrm{FA}) / \mathrm{P}(\mathrm{SA})$ for the algorithm of [5].

typically turns out to be the most interesting case, since the cases $d_{\min }=0 \mathrm{nmi}$ and $d_{\min }=10 \mathrm{nmi}$ correspond to extreme situations in which either a conflict almost always occurs or there is a negligible number of conflicts, respectively. Setting $P=0.85$ leads to the values of $P(F A)$ and $P(S A)$ reported in Table 3 . We are currently working on a sensitivity analysis of the dependence of $P$ on the flight plans to allow us to choose appropriate values for $P$ for the typically encountered configurations. Other aspects that should be taken into account in this process are the detection of conflict a certain amount of time before it occurs and the prediction of its occurrence time. These aspects highly influence the effectiveness of a prediction/resolution scheme involving the human-in-the-loop component.

\section{Concluding Remarks}

We presented a randomized algorithm for mid-range conflict detection in an ATMS. The key points of our approach are:

1. the explicit separation between the probabilistic models used for prediction and validation, and

2. the use of randomized algorithms to manage the computational complexity of the problem and to provide quantitative estimates of the level of approximation involved.

\begin{tabular}{|c|c|c|c|c|}
\hline \multirow{2}{*}{$\phi$} & & \multicolumn{3}{|c|}{$t_{\min }$} \\
\cline { 3 - 5 } & $d_{\min }$ & 8 & 12 & 20 \\
\hline 30 & 5 & $0.24 / 0.64$ & $0.30 / 0.76$ & $0.31 / .82$ \\
\hline 45 & 5 & $0.19 / 0.75$ & $0.22 / 0.80$ & $0.22 / 0.80$ \\
\hline 90 & 5 & $0.21 / 0.71$ & $0.21 / 0.90$ & $0.28 / 0.89$ \\
\hline
\end{tabular}

Table 3: $\mathrm{P}(\mathrm{FA}) / \mathrm{P}(\mathrm{SA})$ with $\mathrm{P}=0.85$ for our algorithm.

Besides developing methods for choosing the conflict detection threshold, we are currently working on formulating algorithms for conflict resolution for multiple aircraft, using model predictive control techniques and stochastic optimization. In parallel, we are working towards a methodology for formally evaluating the safety properties of the proposed algorithm. This will hopefully lead to a more general probabilistic verification methodology for hybrid systems.

\section{References}

[1] Heinz Erzberger, Thomas J. Davis, and Steven Green, "Design of center-tracon automation system", in Proceedings of the AGARD Guidance and Control Syposium on Machine Intelligence in Air Traffic Management, 1993, pp. 11.1-11.12.

[2] Radio Technical Commission for Aeronautics, "Minimum operational performance standards for traffic alert and collision avoidance system (TCAS) airborn equipment", Tech. Rep. RTCA/DO-185, RTCA, September 1990, Consolidated Edition.

[3] D. Bertsimas and S. Stock Patterson, "The air traffic flow management problem with enroute capacities", Operations Research, vol. 46, pp. 406-422, 1998.

[4] P.B.M Vranas, D. Bertsimas, and A.R. Odoni, "Dynamic gound-holding policies for a network of airports", Transportation Science, vol. 28, pp. 275-291, 1994.

[5] Russell A. Paielli and Heinz Erzberger, "Conflict probability estimation for free flight", Journal of Guidance, Control and Dynamics, vol. 20, no. 3, pp. 588-596, 1997.

[6] L. Yang and James Kuchar, "Prototype conflict alerting logic for free flight", Journal of Guidance Control and Dynamics, vol. 20, no. 4, pp. 768-773, 1997.

[7] Claire Tomlin, George Pappas, and Shankar Sastry, "Conflict resolution for air traffic management: a case study in multi-agent hybrid systems", IEEE Transactions on Automatic Control, vol. 43, no. 4, pp. 509-521, 1998.

[8] G. Chen, G. Chen, and S. Hsu, Linear stochastic control systems, CRC Press, Inc., 1995.

[9] M. Vidyasagar, A theory of learning and generalization: with applications to neural networks and control systems, Springer-Verlag, London, 1997.

[10] James K. Kuchar, A Unified Methodology for the Evaluation of Hazard Alerting Systems, PhD thesis, Massacusetts Institute of Technology, 1995. 